\title{
Towards a better understanding of mouse and human diseases- International Mouse Phenotyping Consortium
}

\author{
QI Xin \& GAO Xiang* \\ MOE Key Laboratory of Model Animal for Disease Study, Model Animal Research Center, Nanjing University, Nanjing 210061, China
}

Received August 4, 2014; accepted December 15, 2014

Citation: Qi X, Gao X. Towards a better understanding of mouse and human diseases-International Mouse Phenotyping Consortium. Sci China Life Sci, 2015, 58: 392-395, doi: 10.1007/s11427-015-4841-4

With the completion of the human genome sequence, the last several decades have seen the pursuit of gene function in vivo. The mouse is generally considered the best model animal for examining gene function because of its high productivity, pure genetic background, and degree of similarity to the human genome. With the development of several gene manipulation methodologies, mice are universally used to study genetics and human diseases. There are more than 20,000 protein-coding genes in the mouse genome. The International Knockout Mouse Consortium (IKMC) project was started to generate a knockout of every protein-coding gene in embryonic stem (ES) cells using standard knockout targeting strategies. Following completion of this project, the International Mouse Phenotyping Consortium (IMPC) was established, with the aim of turning these ES cells into live mice, and exploring their potential defective phenotypes using a standard phenotyping pipeline. Globally, about 20 mouse clinics from Europe, the USA, Canada, Australia, and Asia participated in this 10-year project, in which Model Animal Research Center of Nanjing University is the only one from mainland China. The combined data from these centers will represent a new data platform providing a gene function encyclopedia, and more importantly, a comprehensive picture of the whole genome.

Annotation of the human genome has identified over 20,000 protein-coding genes. Despite the dramatic increase in our knowledge of the human genome, the normal func-

*Corresponding author (email: gaoxiang@nju.edu.cn) tion of many genes remains unknown, or is only predicted based on sequence analysis alone. Making full use of the complete human genome sequence requires the collection of much more data, and the availability of comprehensive biological resources could assist with this process [1].

The mouse is an important model animal for assessing mammalian gene function, as processes involved in development, physiology, and metabolism are similar to their human counterparts. Genetic studies in mice, most of which use gene targeting in ES cells, are traditionally performed by individual laboratories and are focused on a particular field of interest, often leaving gene function partially unexplored. To overcome such disadvantages, a comprehensive analysis of a corresponding knockout mutant mouse line should be carried out. The IMPC was established to assess the function of unknown genes, and to carry out high throughput screening of gene-targeted mutant mice. With more than 20 tests covering multiple important physiological processes, the IMPC phenotyping pipeline has been scientifically validated, and is capable of discovering novel functions of existing or completely unknown genes. To date, multiple phenotypes have been discovered for a large number of gene-targeted mouse strains, revealing the value of screening all gene mutants for a wider range of traits [2]. The ultimate aim of the IMPC is to provide the scientific community all the data and mice resource for better understanding of gene function and further investigation.

The plan of IMPC requires a long-term (10-year) commitment and will be carried out in two phases: 
Phase I (2011-2016): Complete and assess existing programs (e.g., EUMODIC), build all the production and phenotyping centers and determine the capacity of each, create a central database, then complete standardized production and phenotyping of 5,000 targeted genes.

Phase II (2016-2021): Assess performance of Phase I, adjust pipelines and operations as necessary, and scale operations to complete standardized production and phenotyping of the other 15,000 targeted genes in the genome (cited from IMPC business plan).

Further details of the IMPC project will be discussed briefly below.

(i) ES cell resource. The IKMC, which combines the European Conditional Mouse Mutagenesis Programme (EUCOMM) and the Knockout Mouse Project (KOMP), has completed more than 10,000 targeted ES cell mutations of protein-coding genes [3]. To study gene functions, IMPC makes use of this knockout ES cell collection, which is based on the "knockout-first" (tm1a) allele [4]. The knockout-first allele contains an in-frame fusion of lacZ and neo cassettes, with T2A peptide sequences included to allow independent expression of lac $Z$ and neo, respectively (Figure S1 in Supporting Information). With an engrailed 2 gene (En2) splice acceptor sequence and poly-A transcription termination signals that disrupt the function of the targeted gene, the tm1a allele ensures gene disruption, while expression of $l a c Z$ is under the control of the endogenous promoter that would designate gene expression during development. Exposure to Cre recombinase converts tmla to the tm $1 \mathrm{~b}$ allele, generating a conventional null-allele with $l a c Z$ tagged. By crossing the tmla allele with the Flp transgenic tool strain, it is turned into a tm1c conditional allele without lacZ. For the IMPC project, the tm1b null allele will be phenotyped, but only the tmle deletion allele is available for certain genes.

(ii) Mouse production. Mice homozygous for the tm1b or tm1e null allele will be generated and entered into the adult phenotyping pipeline. For mice that are homozygous lethal, embryos and heterozygous adults will be phenotyped instead. Production of mice from ES cells and then breeding of chimera cohorts to heterozygous $\mathrm{Cre}^{+/-}$animals, or heterozygotes to homozygotes, is very time-consuming and a major rate-limiting factor for high-throughput phenotyping. Ideally, seven pairs of mutant mice of each sex will pass through the pipeline at the same time and same age. However, the breeding required to provide complete full-sized cohorts is difficult, and mice are often sent to the pipeline in smaller cohorts. In these cases, data can be achieved from two sequential test groups. Statistical analysis determined that for most tests, seven pairs of mice would yield the optimal number of detected parameters per mouse.

(iii) Standard operating procedures (SOPs). SOPs cover the equipment, consumables, quality control, and the protocols to deliver robust, validated, phenotyping outcomes. Effective SOPs ensure that results are comparable within and between different laboratories and over time. The IMPC standard SOP is derived and briefed from the EMPReSSlim pipelines [2].

For all 18 mandatory tests and the additional nonmandatory tests now applied in the IMPC pipeline (Figure $1)$, the critical area of pipeline operation is to ensure that each test will not influence the results of the next. In the IMPC project, the appropriate order of tests performed was carefully considered. This is particularly necessary for behavioral tests, where handling has potential effects on performance. However, individual centers are encouraged to add additional tests to the IMPC pipeline, on condition of not influencing time points or the data generated from the IMPC tests.

A key factor in the design of the pipeline is that all tests are high-throughput. The IMPC pipeline covers a diverse range of biological systems. Using the 18 mandatory tests, the following physiological systems could be screened for primary defects with the listed tests:

Neurology: SHIRPA test, open field, pre-pulse inhibition (PPI), brain anatomy

Cardiovascular: echo, ECG, blood clinic chemistry, heart weight, heart histology

Metabolism: body weight curve, food intake/energy expenditure, fat/lean composition, glucose tolerance test, blood clinic chemistry

Senses: auditory brain stem response, slit lamp, eye fundus

Musculoskeletal: grip strength, X-ray, bone mineral density/content

Immune: blood cell count, blood/spleen cell profile by flow cytometry

Development: embryo LacZ staining, viability at E12.5 and other time points

For embryonic lethal lines, embryonic phenotyping is necessary. The design of the tmla allele, with the inclusion of a lacZ reporter in the construct, allows analysis of embryonic and adult gene expression patterns. The annotation of expression patterns for the genes analyzed will add more information on the expected gene function.

The time of embryonic death is assessed by imaging in vivo or by dissecting embryos at different stages of development. An expression pattern of each gene during embryonic development is also required to generate more information on gene function during embryonic development.

(iv) Data output. The data coordination center (DCC) is built to ensure that the data generated from production and phenotyping centers are captured, validated, and subjected to quality control prior to being deposited into the publically accessible data center [5].

One component of the DCC is iMits, a database and web portal that efficiently administers the phenotyping data from all of the individual mouse laboratory information management system (LIMS) from each of the participants. In the iMits database each center selects the gene of interest 


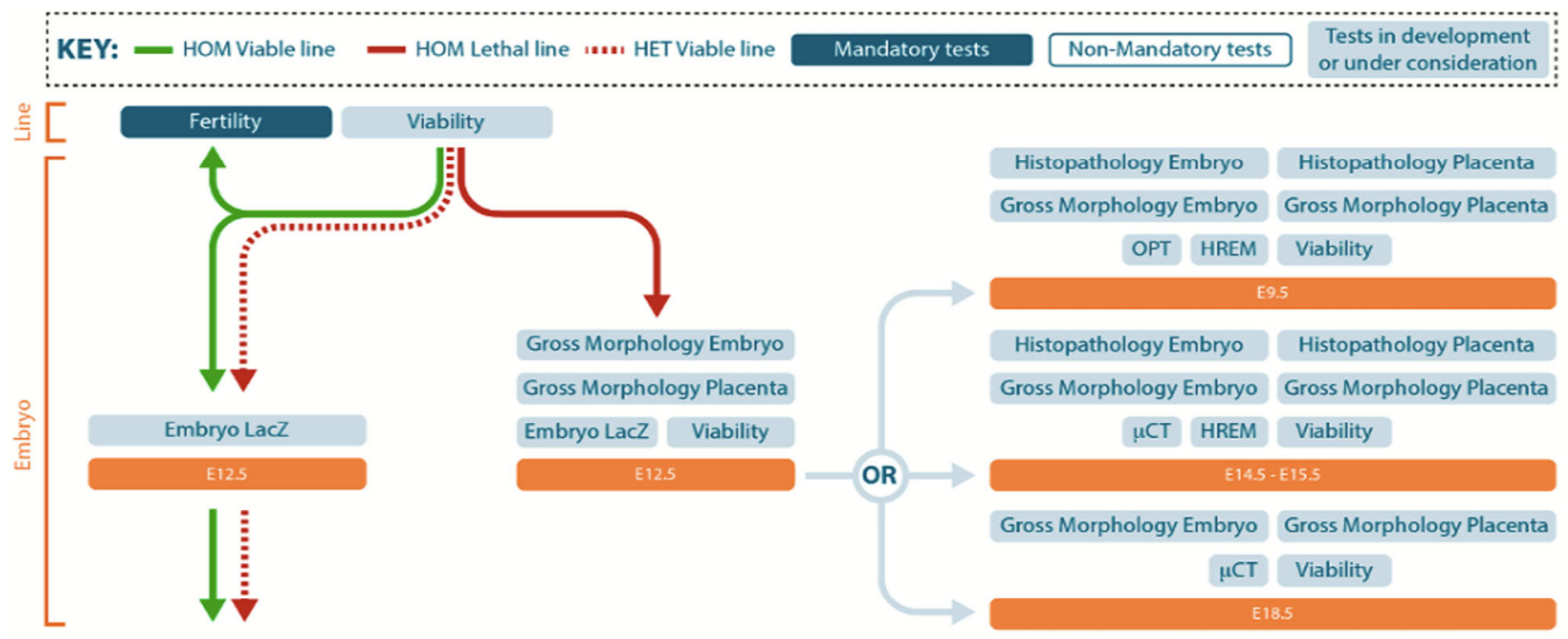

$7 \mathrm{M}+7 \mathrm{~F}$ Mutant Adult Mice

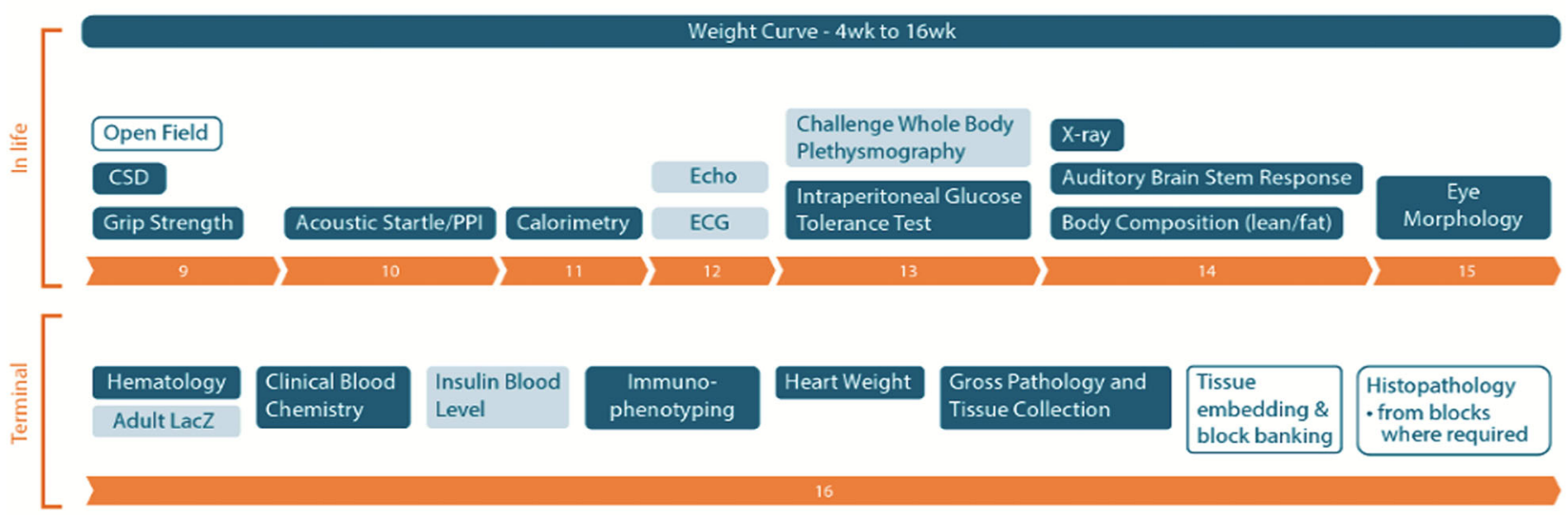

Figure 1 IMPC phenotyping pipeline (adapted from website https://www.mousephenotype.org/impress). The pipeline includes testing of adults from 9-16 weeks of age, as well as embryonic tests at different stages.

and then provides mouse production and phenotyping progress information for that gene. iMits summarizes the reports on total production and pipeline efficiency for each center, and monthly reports of activity. The progress of each gene is publicly accessible from the IMPC portal. For phenotyping data, a common XML data format is used to exchange diverse data from local LIMS into a standard format, and then automatically uploaded onto a local FTP server. The data format defines required data for each individual mouse, including mouse ID, zygosity, sex, strain background, whether or not the mouse is a control mouse, date of birth, and the phenotyping data generated for the specific mouse with uniform units. After being captured into the DCC, these primary data, including bone mineral density, plasma calcium concentration, and any abnormalities in the eye morphology, are analyzed by an annotation pipeline that compares data to a standard range to define any false data resulting from equipment breakdown or inappropriate unit transformation. Correct data from each of the mutant lines is then compared with the baseline strain to identify statisti- cally significant deviants. After all data for a gene mutant mouse strain are captured and validated, the phenotyping status of the particular gene is updated to "complete" on iMits. All gene data and additional information will be available on the IMPC portal, which provides a single point of access to all IMPC data and a variety of tools to search and view these data.

To date, almost 2,000 genes have been completed as part of the IMPC phase I project. Many more genes are in the process of mouse production and phenotyping. The IMPC phenotyping pipeline is always improving, and there will be a constant development process whereby new and improved tests are added to provide additional functional information on the genome. In addition, the gene mutation techniques are also improving. Recently, the CRISPR/Cas9 strategy [6] has proven to be better than the conventional method in terms of time and cost involved for generating gene mutant mouse strains. Although this method is still being developed and has not been applied in large-scale experiments, it is likely to be a potent tool in the future. 
1 Brown SD, Hancock JM, Gates H. Understanding mammalian genetic systems: the challenge of phenotyping in the mouse. PLoS Genet, 2006, 2: e118

2 White JK, Gerdin AK, Karp NA, Ryder E, Buljan M, Bussell JN, Salisbury J, Clare S, Ingham NJ, Podrini C, Houghton R, Estabel J, Bottomley JR, Melvin DG, Sunter D, Adams NC, Sanger Institute Mouse Genetics Project, Tannahill D, Logan DW, Macarthur DG, Flint J, Mahajan VB, Tsang SH, Smyth I, Watt FM, Skarnes WC, Dougan G, Adams DJ, Ramirez-Solis R, Bradley A, Steel KP. Genome-wide generation and systematic phenotyping of knockout mice reveals new roles for many genes. Cell, 2013, 154: 452-464

3 Skarnes WC, Rosen B, West AP, Koutsourakis M, Bushell W, Iyer V, Mujica AO, Thomas M, Harrow J, Cox T, Jackson D, Severin J, Biggs P, Fu J, Nefedov M, de Jong PJ, Stewart AF, Bradley A. A conditional knockout resource for the genome-wide study of mouse gene function. Nature, 2011, 474: 337-342

4 Ryder E, Doe B, Gleeson D, Houghton R, Dalvi P, Grau E, Habib B, Miklejewska E, Newman S, Sethi D, Sinclair C, Vyas S, Wardle-Jones H, Sanger Mouse Genetics Project, Bottomley J, Bussell J, Galli A, Salisbury J, Ramirez-Solis R. Rapid conversion of EUCOMM/KOMP-CSD alleles in mouse embryos using a cell-permeable Cre recombinase. Transgenic Res, 2014, 23: 177-185

5 Mallon AM, Iyer V, Melvin D, Morgan H, Parkinson H, Brown SD, Flicek P, Skarnes WC. Accessing data from the International Mouse Phenotyping Consortium: state of the art and future plans. Mamm Genome, 2012, 23: 641-652

6 Zhang L, Zhou Q. CRISPR/Cas technology: a revolutionary approach for genome engineering. Sci China Life Sci, 2014, 57: 639-640

Open Access This article is distributed under the terms of the Creative Commons Attribution License which permits any use, distribution, and reproduction in any medium, provided the original author(s) and source are credited.

\section{Supporting Information}

Figure S1 Design of the knockout first allele employed by IMPC, adapted from [3].

The supporting information is available online at life.scichina.com and link.springer.com. The supporting materials are published as submitted, without typesetting or editing. The responsibility for scientific accuracy and content remains entirely with the authors. 